\section{INBRX-121 IS AN NKP46-TARGETED DETUNED IL-2 WITH ANTITUMOR ACTIVITY AS A MONOTHERAPY OR IN COMBINATION WITH MULTIPLE CANCER IMMUNOTHERAPY MODALITIES}

Florian Sulzmaier*, Heather Kinkead, Anya Polovina, Nadja Kern, Angelica Sanabria, Chelsie Macedo, Abrahim Hussain, Sae Jeong Ahn, Rajay Pandit, William Crago, John Timmer, Analeah Heidt, Brendan Eckelman. Inhibrx Inc., La Jolla, CA, United States

Background Natural Killer (NK) cells play a pivotal role in cancer immunosurveillance due to their potent cytolytic activity and NK cell-centric therapies have emerged as safer alternatives to targeting $\mathrm{T}$ cells. ${ }^{1}{ }^{2}$ Interleukin 2 (IL-2) drives NK cell expansion and activity, but its therapeutic utility is limited by rapid clearance, expansion of immunosuppressive regulatory $\mathrm{T}$ cells, and by severe dose-limiting toxicities. ${ }^{3}$ INBRX-121 overcomes these liabilities through specific targeting of an affinity-detuned IL-2 variant to cells expressing NKp46.

Methods An IL-2 variant was engineered to eliminate binding to CD25 and to have attenuated affinity for CD122. This detuned cytokine was fused to a high-affinity single-domain antibody targeting NKp46 to generate INBRX-121. The ability of INBRX-121 to target IL-2-like signaling specifically to NKp46-expressing cells was evaluated in vitro using human lymphocytes by measuring STAT5 signaling and cytotoxic activity in tumor cell co-cultures. Characterization of the pharmacokinetic/pharmacodynamic relationship of INBRX-121 was completed in non-human primates across escalating dose levels, while anti-tumor activity as a monotherapy and in combination with Rituximab or PD-1 checkpoint blockade was tested in Raji xenografts and syngeneic CT-26 mouse models, respectively.

Results INBRX-121 induces a STAT5 signal equal to that of wild-type IL-2 in human lymphocytes but shows an NK cellcentric activity profile. Cells targeted by INBRX-121 have increased proliferative capacity and improved cytotoxicity in antibody-dependent and -independent tumor cell killing assays. INBRX-121 shows prolonged pharmacokinetic exposure in vivo and is well-tolerated in mice and cynomolgus monkeys. The NKp46-specific IL-2 stimulus in these models results in a robust, dose-dependent NK cell expansion. As predicted by its in vitro activity, INBRX-121 also enhances the cytotoxic capacity of NK cells in vivo measured via elevated intracellular levels of Granzyme B. In a Raji xenograft model, INBRX121 slows tumor growth as a single agent and synergizes with Rituximab to induce complete tumor regression. Similarly, cotreatment with INBRX-121 improves the incomplete suppression of CT-26 tumor growth by a PD-1 blocking antibody to yield complete responses that show immunological memory upon re-challenge.

Conclusions INBRX-121 offers a unique approach to overcoming the limitations of current IL-2 therapeutics. NKp46-targeting of a detuned IL-2 variant helps to avoid IL-2-mediated toxicity while enhancing the antitumor activities of NK cells. Through its novel therapeutic concept INBRX-121 provides a promising treatment option for multiple cancer indications both as a monotherapy and in combination with a variety of frontline agents.

\section{REFERENCES}

1. Shimasaki N, Jain A, Campana D. NK cells for cancer immunotherapy. Nat Rev Drug Discov 2020;19:200-218.

2. Liu S, Galat V, Galat $Y$, Lee $Y$, Wainwright $D$, Wu J. NK cell-based cancer immunotherapy: from basic biology to clinical development. I Hematol Oncol 2021;14:7.
3. Overwijk W, Tagliaferri M, Zalevsky J. Engineering IL-2 to give new life to T Cell immunotherapy. Annu Rev Med 2021;72:281-311.

Ethics Approval All animal studies were conducted in accordance with AAALAC regulations and were approved by the IACUC for Explora BioLabs (\#SP17-010-013) and BTS Research (20-015 Enrollment 05).

http://dx.doi.org/10.1136/jitc-2021-SITC2021.722 